\title{
A Botanist's Cognitive View on Plant Growth: Cross-Talk between Developmental and Sensitivity Networks
}

\author{
Dhananjay K. Pandey, Bhupendra Chaudhary* \\ School of Biotechnology, Gautam Buddha University, Greater Noida, India \\ Email: ^bhupendrach@gmail.com
}

How to cite this paper: Pandey, D.K. and Chaudhary, B. (2016) A Botanist's Cognitive View on Plant Growth: Cross-Talk between Developmental and Sensitivity Networks. American Journal of Plant Sciences, 7, 23072322.

http://dx.doi.org/10.4236/ajps.2016.715203

Received: October 19, 2016

Accepted: November 21, 2016

Published: November 24, 2016

Copyright $\odot 2016$ by authors and Scientific Research Publishing Inc. This work is licensed under the Creative Commons Attribution International License (CC BY 4.0).

http://creativecommons.org/licenses/by/4.0/

\begin{abstract}
An alteration in plant phenotypes assisted by their responses to the environmental stimuli (=tropism) has been fundamental to understand the "plant sensitivity" that plays a crucial role in plants' adaptive success. Plants succeed through the deployment of moderators controlling polar auxin-transport determining organ bending. Stimulus-specific effectors can be synthesized by the outer peripheral cells at the bending sites where they target highly conserved cellular processes and potentially persuade the plant sensitivity at large. Remarkably, the peripheral cells require different time-intervals to achieve the threshold expression-levels of stimulus-specific molecular responders. After stimulus perception, tropic curvatures (especially at growing root-apices) are duly coordinated via integrated chemical and electrical signalling which is the key to cellular communications. Thus, the acquired phenotypic alterations are the perplexed outcome of plant's developmental pace, complemented by the sensitivity. A novel aspect of this study is to advance our understanding of plant developmental-programming and the extent of plant-sensitivity, determining the plant growth and their future applications.
\end{abstract}

\section{Keywords}

Tropism, Anisotropy, Plant Development, Polar Auxin Transport, Phototropic Response, Gravitropic Response

\section{Introduction}

It is believed that plant species have evolved in response to the natural selection and subsequently diversified to different climatic conditions. In this process, certain genetic and epigenetic components were selected and largely inherited by their descendants, 
thus delineating the species developmental silhouette. Since plant species had predominantly evolved sessile in their origins, they do respond to the varying external atmosphere by their altered growth patterns. Such changes in growth patterns are the result of the plant's response to environmental stimuli, which are indeed one of many plant responses to environmental stimuli, colloquially known as the "tropic movements". This phenomenon directly or indirectly endorses the survival of a species under natural selection, and therefore plays a crucial role in the plants' adaptive diversification. In a natural environment, plants face a variety of revelations such as light, gravity, heat, water, mechanical obstacles and others. In result, they do respond to such exposures for better subsistence while maintaining their developmental rhythm. Plants do synchronize with several molecular responders; these are required for natural growth and development. These molecules have evolved for sensing the external stimuli and establishing a fine balance between the innate developmental pace of a plant and modulation in the re-programming of adaptation. In result, cells show altered growth patterns attributed to the plant tropism.

Tropism, an instant response of plants to environmental cues, serves as the foremost mode of regulating the morpho-plasticity of plants in the altered environment. Plants' responses to such environmental cues have been characterized and supposedly contribute to their adaptive success in the altered environment. For example, the evolution of i) stomatal guard cells and their movement, and ii) alteration in the growth pattern of stem and roots. Similarly, plants do encounter various hostile challenges during their life cycles. To adapt to such challenges, the plants acquire many physiological and developmental changes. Empirical studies have focussed on the identification of the indispensable growth processes and their metabolic, chemical, or hormonal basis responsible for temporal developmental pace. Thus, plant tropism has been a fundamental outcome of the plant's response to the external stimuli, often termed as plant "sensitivity". Other contributing factors of cellular turgor may also drive plant movements, adding to the sensitivity factor. Hence, an individual's competence to adapt is the sum of its defined developmental pace and its responses to the perceived stimuli (tropic movements).

Genomic response to the perceived stimuli has been proportional to the endogenous hormonal levels, especially auxin concentrations that largely vary across cellular panels. The hormonal manipulation of the cells specialized with perceived stimulus is oriented to light [1]; touch [2]; gravity [3], and water [4]. However, limited knowledge is available on how the genetic portions accountable for specific auxin-responsive elements show their elevated expression in correlation to the environmental clues, particularly those that might be presumed to have developmental implications, such as directed organ bending? The focus of this review is to synopsize the current information of "plant tropic movements" to understand the regulation of developmentally dynamic plant sensitivity to hormone and environmental signals, and to establish an association connecting developmental programming and the extent of plant sensitivity that should augment the future research of plant tropism and plant sensory biology. 


\section{Implications of Anisotropic Cellular Expansion in Plant Tropism}

Usually, plant cells have relatively rigid cell walls that are tightly joined to the adjacent cell walls maintaining the tissue integrity. The growing plant cells have been assumed to be hyper-rectangles with a defined aspect ratio of the longest side to the shortest side. The well-described developmental pace of plant cells is usually proportional to the acquired cell aspect ratios. This is majorly dependent upon the cell division and their arrangement, thus controlling the cellular geometry during development [5]. Based on the selection of cell division planes, Hofmeister [6] proposed the symmetric cell division largely adopted by the growing cells, which insert the newly developed cell wall at the right angle to the axis. On the contrary, the most efficient cell division could be the formation of the shortest wall during cytokinesis generating two unequal daughter cells, as reviewed by Prusinkiewicz and Runions [29]. This variability in the theory of symmetric cell division had introduced another algorithm of asymmetric expansion of plant cells, especially peripheral or anticlinal cells, primarily postulated in relation to the principal directions of growth (PDGs) [7] [8]. In phototropism, under the influence of phyto-regulators and supported by the concerned influx and efflux carrier proteins, for example, PIN and LAX proteins, these PDGs produce three-dimensional celldeformations for the directional curvature of organs [9].

Such high variability in the dissecting planes may be accounted for random and shortest planes during cytokinesis and might not necessarily follow the rule of symmetric cell division. This novel concept of asymmetric cell division has certainly transformed our outlook towards the dynamicity of cell aspect ratio, cell division, and their orientation. However, relatively less is explicit about the manner in which the determining forces or external signals regulate the already commenced progression of cell division spatially or temporally? In all scenarios, cells are prompted to grow and divide mainly in the PDGs, though with unequal diameters; colloquially defined as "anisotropy". Would these PDGs be considered exceptionally receptive to the external signals for intended cytokinesis? Or, is such anisotropic growth exclusively responsible for the alteration in a typical aspect ratio of cells? In such case, what is the typical aspect ratio of any tissue or organ development in an organism?

Peaucelle et al. [28] profoundly examined the stimulation of cellular asymmetry in the anticlinal walls of growing Arabidopsis hypocotyls by pectin de-methylestrification and microtubule orientation. Authors have focussed on a remarkable outlook of growth symmetry breaking during hypocotyl development and re-orientation of microtubules, which in result transform the isotropic growth into anisotropic growth. Such tropicconversion is a discrete phenomenon that may have led to altered cellular aspect ratio and accelerating the developmental pace. Consequently, Peaucelle et al. [28] identified the requirement of such breaking in the symmetry for acquiring anisotropy during cellular expansion. An example of prompt and noticeable plant anisotropy is the root geotropism under the influence of gravitational force. In theory, such asymmetric cellular 
expansion during gravitropism may be directed by the applied mechanical stresses on the growing cells, mainly through the differential distribution of phyto-morphogens [10]. However, breaking growth symmetry would not be directly linked to the axis growth and developmental pace, which is substantially contributed by the reorientation of cortical microtubules. In the root meristematic tissues, the microtubules get more organized in the vertical orientation of axis with the increased distance from the apicaltip [11]. However, is the alignment of cortical microtubules on the root axis necessary for positive gravitropism? Mutational analysis of BOTERO1 (Bot1) locus which is responsible for anisotropic growth in non-apical meristematic cells showed that Bot1 roots grow under positive gravitropism indicating for the non-significant contribution of ordered cortical microtubules at least in root gravotropism [11]. The developmental pace may get tremendous alterations because the cellular exhibition of the degree of sensitivity at temporal scale determines the future organ development.

Notwithstanding striking discoveries, little is explicit about which of the external stimulus does perturb the developmental pace utmost. Alternatively, what is the degree of plant sensitivity in a manifested environment that is notably manipulates directional growth and cell aspect ratio? Sensitivity driven alterations in the growth pattern usually tend to create tension on the integrated cell wall network. The molecular determinants of anisotropic growth during root gravitropism regulate vesicular trafficking between cellular compartments during the formation of a root-wave by clockwise and anticlockwise curvatures. This important phenomenon is regulated by $R H D 3$ gene, as loss-of-function mutation of the rhd3 gene showed suppression of epidermal cell file rotation, root waving and skewing which are extremely vital events for root gravotropism [12]. Similarly, $M L O 4$ gene predominantly expressed in the tips of growing roots of Arabidopsis and regulate root expansion under thigmotropism [13]. Thus, various site-specific molecular determinants may regulate the bending of target organs, such as roots or stem under a tropic movement (Figure 1(a)). Nonetheless, few questions remain elusive in this field, for example, what would be the genetic control of such phenotypes modifications during altered aspect ratio of the growing cells? Is any particular morphogen, such as auxin or cytokinin prominent during organ bending? What could be the impact of such morphogen on the cell aspect ratio? Who is the principal regulator of PDGs; and what is the distribution of such regulator across cells or tissues during altered growth?

\section{Auxin Concentration Gradient: Polar Transport or Localized Over-Production}

The primer article by Gilroy [34] profoundly emphasized the extraordinarily complex mechanism of plant tropism. The implications of this study are to understand the role of auxin in perceiving the stimuli, and its movement during tropic conversion. Notably, there is an asymmetric flow of auxin among cells usually from inner peripheral layer to the outer peripheral layer and across the direction of organ bending (Figure 1(b)). Therefore, the mechanism of plant tropism is itself sporadic, and potentially persuades 


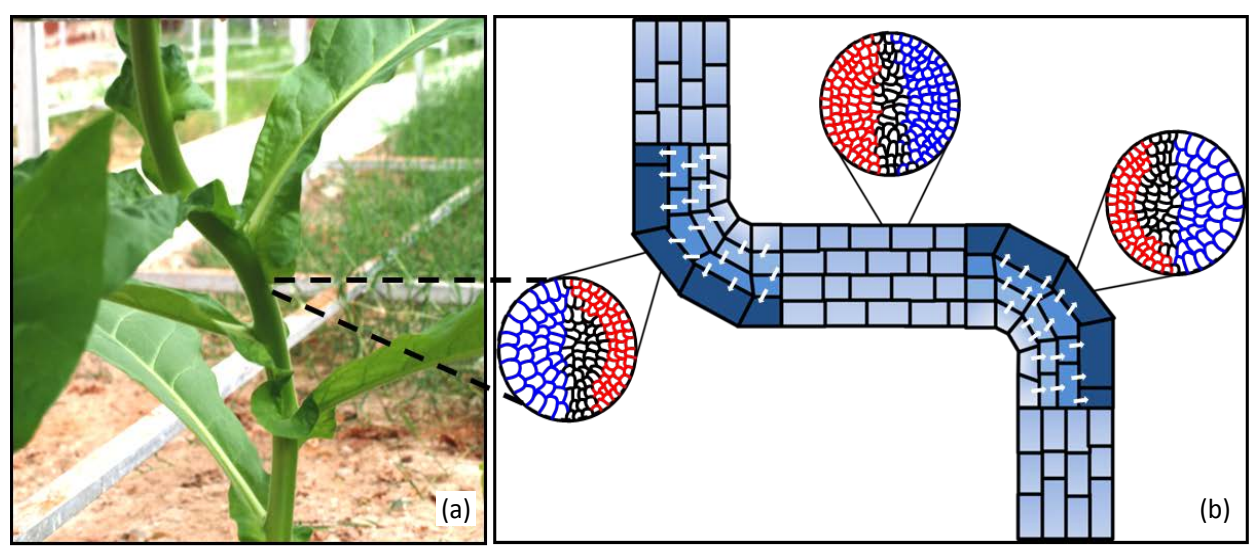

Figure 1. Anisotropic growth of a plant during an environmental stimulus. (a) A tobacco plant is showing directional growth in response to the environmental cues. (b) Polar auxin transportation is prominent in the outer peripheral layer of cells (shown with white arrows) that may initiate the organ bending during cell growth and development. Due to such polar auxin transportation, drastic changes in the aspect ratio of outer peripheral cells occur (marked in blue), having no such change in the middle (marked in black) and inner layer cells (marked in red). Outer peripheral cells on both the bending regions acquire increased auxin concentration, whereas the middle elongating regions are having uniformly distributed auxin.

the plant sensitivity at large. It also provides a fascinating advancement of our understanding of the potential regulation of auxin movement through $\mathrm{pH}$-dependent cellular partitioning, coupled with several uptake transporters and efflux transporters. However, it could be further envisaged for several ensuing experiments using state-of-theart methodologies, for example, performing gene expression and localization analyses of auxin sensors and transporters that are putatively involved in the lateral movement of auxin. It would reveal the precise quotient and interval during the plant's response to the stimuli, and how it varies across stimuli. Kwon et al. [14] used this approach in Arabidopsis to demonstrate the increased expression of MYBH transcription factor and increased auxin biosynthesis during hypocotyl elongation in no light condition. The MYBH transcription factors are highly responsive to the environmental cues, especially to photo-conditions as the mybh mutant lines did not show any differences in the hypocotyl length compared to the wild type in continuous light condition [14].

Besides having multiple roles in various developmental processes, auxin is essential to trigger the plant sensitivity in response to environmental stimuli. Nevertheless, an intriguing question endures whether only auxin transport ensues across layers of a stem or root underlying development or bending. Is auxin transport alone sufficient for supporting organ development or organ-specific tropic responses? On the other hand, is there a peripheral layer-specific increase in the auxin biosynthesis in parallel to auxin transport? For example, during normal root development, auxin is synthesized in the shoot-tips and polarly transported to the root tissues. This process generates the dynamic auxin-gradient across cell layers that is responsible for the plant development [15] [16]. The asymmetric downward distribution of IAA results into the gravitropic curvature of coleoptile growth by the regulation of auxin signaling pathways [17]. Chen 
et al. [18] identified a group of genes through mutant analyses, which regulate auxin biosynthesis in the developing root cells. However, over expression of these auxin biosynthetic genes did not rescue the mutant defects in roots. From a developmental perspective, it is interesting to note that only auxin transport from shoots to roots is not sufficient for supporting normal root development, especially for desired tropic movements, but also requires assistance from localized auxin over-production [17] [18].

Moreover, exploring the specific functions and mechanisms of cellular diffusion of auxin by its sensors and transporters, further insight into the localized production of auxin in the peripheral cells because of plant sensitivity is probably unexplored. Presently, our understanding of the localized production of auxin in the anticlinal cells remains elusive, which could now be, achieved with the advent of genomics era using global approaches such as NGS platforms. By using outer and inner peripheral cells as tissues, these platforms would identify differential expression analyses of all genes involved in organ bending because of plant sensitivity. However, targeting the peripheral cells for gene expression analysis underlying responses to any particular stimulus might be challenging. Innovative technologies for harvesting the cellular layers, such as laser micro-dissections of both outer and inner peripheral layers could be of remarkable evidence.

\section{Pre- and Post-Polar Auxin Transport Intervals}

Now, it is widely assumed that anisotropic growth of an organ is due to uneven distribution of auxin across cellular layers termed as polar auxin transport (PAT). The PAT is unidirectional, firmly controlled, and largely dependent on the presence of efflux carriers across plasma membrane for auxin trafficking [19] (Figure 2). Targeted efforts have clearly identified mechanisms and moderators controlling polar auxin redistribution resulting in the tropic movements, for example, AUX1 influx carriers, PIN efflux carriers, and P-GLYCOPROTEIN transporters. Are such PAT-moderators stimulusspecific or global? What does the expression of these moderators depend on across external stimuli? How much time is required by the target cell prior to instigating the expression of such moderators? Or what is the degree of plant sensitivity among different stimulus-specific response systems?

It is assumed that pre-PAT mechanisms take a short interval, whereas post-PAT processes such as altered cell division and elongation against innate developmental pace are long processes; and thus, may vary across stimulus types (Figure 2). Is the time consumed by the post-PAT mechanisms equal across tropic movements? Or, is it influenced by the magnitude of a stimulus? The regulation of these auxin modulators usually depends on the degree of stimuli that consecutively induce plant sensitivity. The phosphorylated PIN efflux carriers have been identified to play the key roles in redirecting the intracellular auxin and maintaining the required auxin concentration in the target tissue. The acquired auxin concentration may vary across tissue types; or in the same tissue in a different environment, thus competing with plant's developmental pace. 


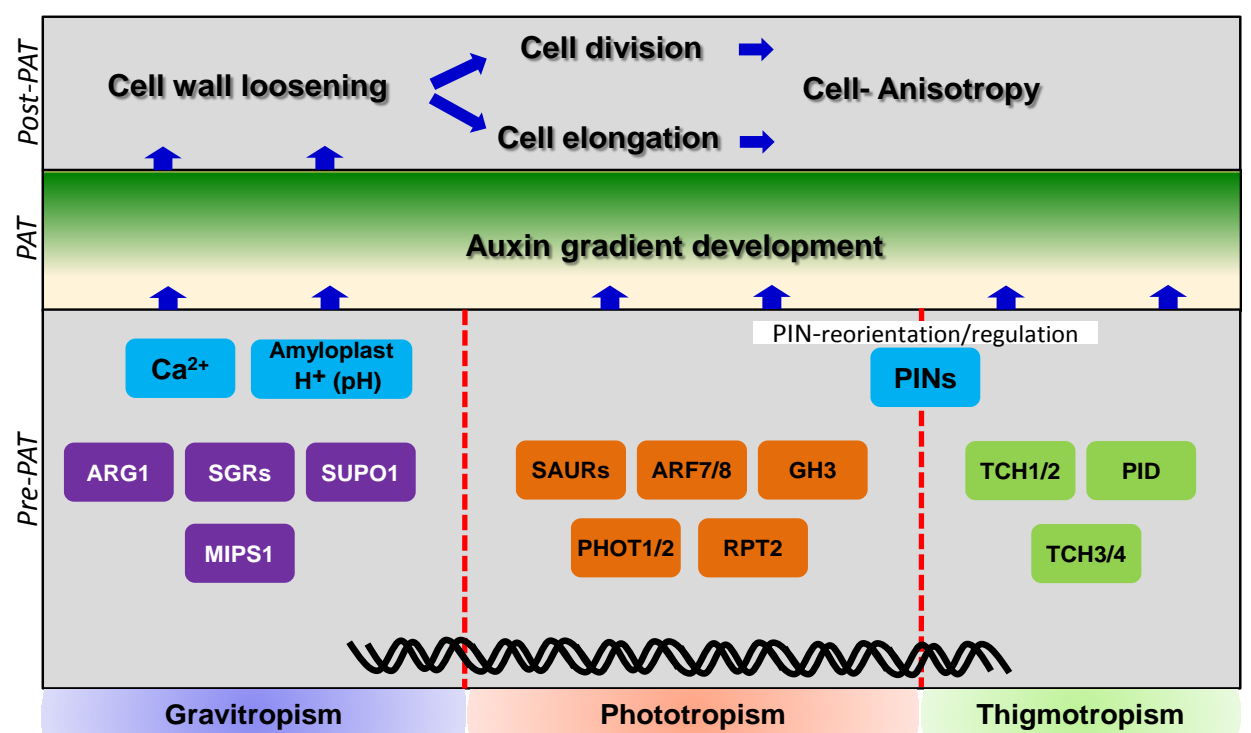

Figure 2. The molecular interplay of key effector molecules under different tropic movements, prior to the establishment of an auxin gradient in the outer peripheral cells. After the perception of a stimulus, the effector molecules are produced rapidly and interact with each other. Various molecules are unique to a specific stimulus, however, certain molecules are conserved across response-to-stimulus systems. For example, PINs are common factors between phototropism and thigmotropism; as well as $\mathrm{Ca}^{+}$are abundantly available as a secondary messenger in both gravitropism and phototropism. These effector molecules help in the establishment of auxin gradient in the cell (marked in green) mainly by the polar auxin transport and by localized production in the peripheral cells. In result, it helps in cell division and elongation principally by the loosening of the cell wall, which is the basis of anisotropic growth of outer peripheral cells and contributes to the organ bending.

Small auxin-up RNAs (SAURs), a class of auxin-responsive genes have been identified symmetrically distributed across layers of vertically elongating hypocotyl and epicotyl tissues. However, SAURs show their asymmetric distribution under gravitropism in the horizontally grown hypocotyls (Figure 2). Notably, re-distribution of SAURs is extremely prompt compared to the developmental pace of the target tissues, guiding the cell elongation. Moreover, the gravitational signals are extraordinarily perceived and magnified during the pre-PAT period through additional factors, namely ARG1, SGRs, SUPO, MIPS1 and establishing an auxin gradient (Figure 2). Similarly, phototropin 1 (PHOT1) paralog regulates perception and amplification of light signals upon ubiquitination through a profound interaction with NPH3 and CUL3/CRL3 molecules (Figure 2). Whereas during the commencement of PAT mechanism, secondary messengers $\mathrm{Ca}^{2+}$ regulated by the pertinent TCHs/PID effectors and NHR1/RHY4 are responsible for thigmo-signal perception and hydrotropism, respectively (Figure 2). Similarly, several factors have already been characterized for their involvement in different stimulus-response systems and are summarized in Table 1.

\section{Polar Auxin Transport and Plant Sensory System}

During normal root growth in soil, the robust and reproducible apical root cap protects 
Table 1. Molecular factors characterized for their application in different stimulus-response systems acquired spatially or temporally during plant development.

\begin{tabular}{|c|c|c|c|c|}
\hline Plant response & $\begin{array}{l}\text { Tissue/response } \\
\text { availability }\end{array}$ & Molecular effectors/responders & Functions & References \\
\hline Phototropism & $\begin{array}{l}\text { Root - } \\
\text { Shoot }+\end{array}$ & $\begin{array}{l}\text { PHOT1, PHOT2, NPH1, NPH3, RPT2, ARF7, ARF8, } \\
\text { IAA19/MSG2, GH3, SAUR gene families, SCF-TIR1, } \\
A B C B 19 \text { and } A U X 1, C R Y 1 \text {, and } C R Y 2, \mathrm{PKS} 1, \mathrm{PKS} 2 \text {, } \\
\text { and PKS4, ABP1 and TIR1/AFB receptors, TSI, DFL1, } \\
P H Y A, C U L 3, E H B 1, R C N 1\end{array}$ & $\begin{array}{l}\text { Establishment of auxin gradient; } \\
\text { Cell wall loosening and cell elongation; } \\
\text { Anisotropic growth }\end{array}$ & $\begin{array}{l}{[1][14]} \\
{[20]-[30]}\end{array}$ \\
\hline Gravitropism & $\begin{array}{l}\text { Root }+ \\
\text { Shoot - }\end{array}$ & $\begin{array}{l}\text { Amyloplasts, actin, } \mathrm{Ca}^{2+} \text { ions, Inositol-1,4,5-triphosphate } \\
\text { (IP3), Changes in } \mathrm{pH} \text { due to fluxes in protons (H+), PIN } \\
\text { family, ARG1, SGRs, SUPO1, MIPS1, PIPs, DAG, AUX/ } \\
\text { LAX influx carrier, AGR/PIN, MDR-like efflux carrier, } \\
\text { GRV2, ZIG, ARL2, GPS, TOC132, TOC75, ACTIN2, } \\
\text { FtsZ3, ADK1, ROS, MAPK, PGM1, LPA1, PPLA-I, } \\
\text { WAV3, ACS7, EHB1 }\end{array}$ & $\begin{array}{l}\text { Regulation of auxin transportation; } \\
\text { signal amplification; } \\
\text { Anisotropic growth }\end{array}$ & $\begin{array}{l}{[3][8][19]} \\
{[20][21][23]} \\
{[28][31]-[39]}\end{array}$ \\
\hline Thigmotropism & $\begin{array}{l}\text { Root }+ \\
\text { Shoot }+\end{array}$ & $\begin{array}{l}\mathrm{TCH} 1, \mathrm{TCH} 2, \mathrm{TCH} 3, \mathrm{TCH} 4, \mathrm{PID}, \\
\text { Calmodulin protein, } \mathrm{Ca}^{2+}\end{array}$ & $\begin{array}{l}\text { Regulation of phosphorylation; } \\
\text { breaking of xyloglucan chain; } \\
\text { loosening of the cell wall; } \\
\text { regulation of PIN efflux regulators; } \\
\text { Regulation of auxin transportation; } \\
\text { Anisotropic growth }\end{array}$ & $\begin{array}{l}{[23][24]} \\
{[32][40]}\end{array}$ \\
\hline Hydrotropism & $\begin{array}{l}\text { Root }+ \\
\text { Shoot - }\end{array}$ & Calcium ion, NHR1, RHY4 & $\begin{array}{l}\text { Water potential gradient development; } \\
\text { Regulation of auxin transportation; } \\
\text { Anisotropic growth }\end{array}$ & [41] [42] \\
\hline
\end{tabular}

the tender meristematic cells from the mechanical injuries. Nevertheless, root cap is the most sensitive sensory organ of plant system, transmitting signals promptly to the subapical "transition zone". In result, it regulates cell polarity and tropic movements of roots sensing the gravity, light, water or nutritional components [43]. The root transition zone performs both sensory and motoric processes instantly after sensing the stimulus [44] [45] [46] [47]. A flexible basal border of transition zone initially responds to the gravity stimulus by quick re-arrangement of F-actin filaments of the elongation zone, which accelerate the root epidermal expansion causing root-bending along with the gravitational force [48]. However, for such anisotropic cell expansion, desired concentration of PAT is required to induce gravitropic curvatures [49]. The tropic curvatures of growing root apices are coordinated at very fine-scale by the sensory-motor integrations which is the key to cellular communications. Thus, the PAT-based phenotypic transformations is reliant to the plant sensory systems, and consequently assisting adaptive responses.

During PAT, numerous molecular responders amongst various tropic movements support the transportation of auxin molecules vertically and horizontally across cell walls and membranes. Auxin-binding protein 1 (ABP1) is transported to the outer surface of the plasma membrane via endoplasmic reticulum and subsequently binds with auxin. Upon binding of ABP1 with auxin, it triggers the inhibition of endocytosis, activation of Rho GTPases, and electric responses [50] [51] [52] [53]. The latter may in- 
duce physicochemical alterations in the plasma membrane via endocytic vesicle recycling process and experimented with surprising similarities in the both plant synapses and human brain [44] [50] [54]. Simultaneously, plant-specific PIN proteins also regulate PAT [55] [56] which is essential for plant growth [20], and plant tropism by sensory-motor integrations [45] [55] [56]. Amongst numerous PIN proteins expressed in the roots, PIN2 plays a key role in the root gravitropism through regulation of auxin flow in synapses formed in the transition zone of root apices as the pin 2 mutant shows no bending at the transition zone during an external stimulus [45]. The novel concept of a cell-to-cell communications in plants through synapses formation resembles the immunological synapses characterized in the animal system. These plant molecules are analogous to the neuronal molecules in animals and considered to constitute neuronal synapses, for example, the resemblance of growing root hairs with neuronal axons and Spastic Paraplegias [45] [57]; and evolution of plant synapses in response to a filamentous fungal pathogen or other parasitic attack [43].

A research focus by Baluska et al. (2010) proposed an emerging concept of PAT by neurotransmitter-like secretion of auxin via plasma membrane vesicle trafficking at the root apices [44]. The synapse formation at the base of the transition zone of the root apex in the course of gravitropism directs neurotransmitter-like mode of auxin transport through the process of vesicle recycling. Notably, the neurotransmitter molecules in the animal system and auxin molecules in the regulation of root architecture are conserved analogous tryptophan-derivatives [58]. It has advanced our knowledge of auxin molecules beyond their conventional role as phytohormone in plant growth as neurotransmitter-like molecule based on its biochemical nature and actions as a morphogen in plant morphogenesis and cell to cell transport [59]. The latter has high resemblance with the neurotransmitter-based cellular interaction and transmission through vesicular trafficking at synapses [60]. In tropic movements, especially in gravitropism and phototropism, however, the direction and sensitivity of PAT to various stimuli is essential for anisotropic cellular expansion [61] [62]. The polar transport of auxin in response to different external stimuli, thus reveal very high similarities with synaptic transmissions in animals. The fundamental evidence of synapse formation in root apices postulates important clues to numerous elusive questions in the field and open new avenues for the identification and characterization of neuronal-like features across tissues perceiving tropic curvatures [54]. In addition, characterization of key auxin carrier molecules present on the axis of polarity; and spatial auxin-inducible gene expression cascades would highlight their roles in the progression of different tropisms.

\section{Integrated Viewpoint of Plant Sensitivity}

An interesting and new prospect of a species' adaptive success is to understand about diverse mechanisms and factors assisting innate plant developmental programming independently and parallelly in a coordinated fashion. Here we focus on the developmental adaptation of an individual under a diverse array of stimuli. It is very interesting

to discuss and elaborate how an individual maintains its sensitivity level and endures 
with different stimuli. In addition, what is the extent of acquired sensitivity under a particular stimulus? Is it similar across stimulus-response systems? Remarkably, plants do share independent stimulus-response systems i) across stimuli in the same individual, and ii) similar factors across individual/species for any particular stimulus. Species do have substantial variations at the phenotypic level that contribute to their typical developmental silhouette. But plant sensitivity-driven numerous genetic effectors responsible for altered phenotype are usually conserved among species [63]. For instance, tangential transportation of auxin molecules through PIN, AUX1, WAT1 ABCB1 and ABCB19 channels leads to organ-bending (root or stem). In response to a stimulus, PAT is generally enhanced, but some ambiguity persists in the enormity of native auxin production by the anticlinal cells during stimulus-response system. Therefore, notwithstanding the discrepancies in the plant sensitivity, it is apparent that stimulus-specific key effector molecules or processes are conserved and may provide avenues for future plant developmental biology.

Further, to understand the intricacies of plant sensitivity, it will be essential to explore the molecular basis of the cellular sensing mechanism. The plasma membrane may be the site of action for perceiving the methodically characterized AUX1 influx carriers, PIN efflux carriers, and P-GLYCOPROTEIN transporters. Different stimulusresponse systems have defined key factors responsible for downstream functions, thus regulating plant developmental machinery to embellish growth [21]. As approved by mutant analyses in phototropism, spatial expression analyses of PHOT1 and PHOT2 paralogous proteins in collaboration with RPT2-NPH3 heterodimer elicit complex signaling events in the acquisition of phototropic stimulus-response [22]. Moreover, these molecules in association with PIN protein-channels establish an auxin-gradient in the peripheral cells that maneuver anisotropic growth of the target tissue. Genetic factors such as $T C H$ family genes, $\mathrm{Ca}^{2+}$ and others are involved in the regulation of substrate phosphorylation events, reorientation of PIN family of auxin efflux regulators [23], and eliciting and amplifying the stimulus causing cell wall loosening and cell elongation [24]. Thigmotropism is closely related with gravitropism especially in root tissues and performs their functions/mechanisms in concordance [64].

Interestingly, plant sensitivity in gravitropism is reliant upon gravitational set-point angle (GPSA) which helps in sensing and maintaining gravitropism during plant growth [31]. In roots, altered $\mathrm{pH}$ of cellular milieu through proton fluxes, rearrangement of PIN channel transporters in concordance with cell-starch level and $\mathrm{Ca}^{2+}$ ions regulating IP3 gene level, possibly perceive and amplify the signaling cascade of gravitropism. Do all stimulus-response systems share common effectors during pre- or post-PAT? Is the execution of the perceived signals analogous to stimulus types? During pre-PAT, external signal perceptors, accumulators, and amplifiers are of spatial in nature according to the type of signals, but they widely share the machinery at the inception of post-PAT mechanisms to execute the perceived signals. Interestingly, selective molecules including EHB1 and $\mathrm{Ca}^{2+}$ do share pre-PAT mechanism during light and gravitational stimuli, respectively. Auxin efflux/influx channels including PIN and its 
variants, $\mathrm{AUX1}, \mathrm{ABCB} 1$ and $\mathrm{ABCB} 19$ are the most common and central gateway to control the gradient among different tropic responses for required auxin concentration through PAT (Figure 2).

\section{Developmental Programming versus Magnitude of Plant Sensitivity}

Evidently, there are certain cell signalling molecules that actively respond to the external stimuli, thus controlling the majority of cellular processes. It would be remarkable to determine if the level of intensity of a stimulus is directly proportional to the executive cellular machinery for tropic movement and further to adaptation? Notably, the aerial parts of plants respond to the light promptly and bend towards the light source. For example, hypocotyl tissues grow faster in the dark conditions than seedlings growing in the light; and plants do grow differently in thigmotropism by escaping the mechanical obstacles. Hence, the acquired phenotypic alterations are the perplexed outcome of plant's developmental pace, supplemented with its response to the external stimuli (Figure 3). However, the time taken from a plant to respond to any particular stimulus may vary largely depending upon the type of molecular responders produced in the cell while responding to a stimulus. Selective signaling molecules show expression alterations or are amplified to receive external signals contributing to the organ elongation or bending. For example, TCH4 is abundantly expressed within 30 minutes on the acquisition of touch stimulation but sharply declines afterward [2]. Cytoplasmic $\mathrm{H}^{+}$ion concentration and $\mathrm{pH}$ change are evident within minutes of the gravitropic responses [31]. However, in phototropism, it takes at least 48 - 72 hours for molecular effectors before they could respond at the phenotypic level that may contribute to the growth and development.

Is the magnitude of an external stimulus or plant's response to the perceived stimulus, or both, deciding factors to amend the inherited developmental programming of a

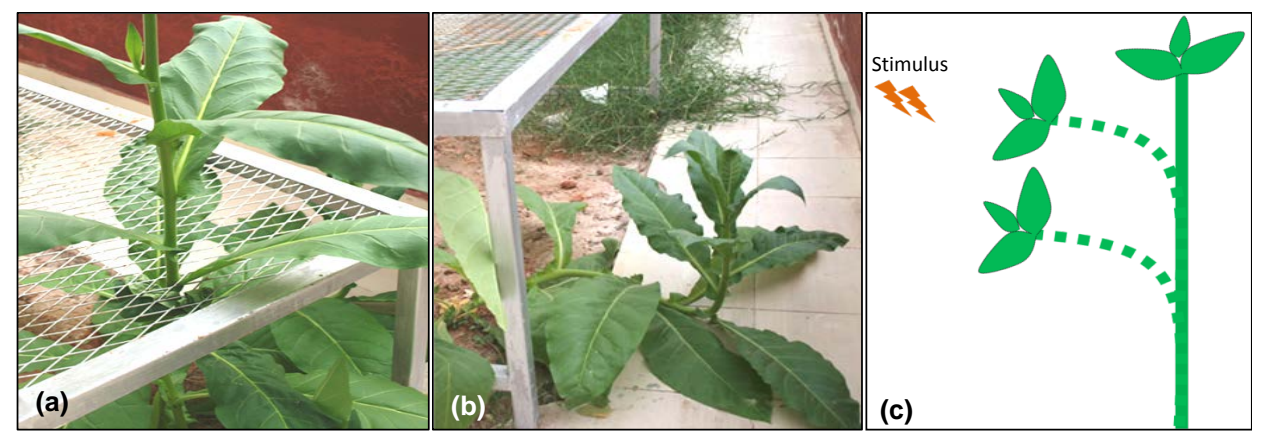

Figure 3. A model of plant growth and development based on the interaction between developmental pace and plant sensitivity. (a) \& (b) The inherited developmental organization of a plant competes with the degree of plant sensitivity towards different atmospheric stimuli during growth. If the developmental pace persists and cellular signaling during PAT is time-consuming, the plant grows as per its developmental programming. (c) The time interval taken by the plant to respond to a stimulus is the deciding factor that defines the path of tropic movement (=bending), thus changes the path of development. 
plant? Certainly, in response to a particular stimulus, the peripheral cells require a time interval to achieve the threshold expression levels of stimulus-specific molecular responders. Thus, it modulates the developmental genetics of a growing cell by a substantial alteration in the transcriptome of the peripheral cells with necessary phenotypic changes. This includes the rearrangement of auxin transporters and gradient establishment that may lead to stimulus-specific tropism. Therefore, we hypothesize that the competitive mechanism of inherited developmental programming and extent of the plant sensitivity determines the future of cellular development. Therefore, different stimulus-response systems, defining spatiotemporal plant sensitivity could be considered as the basis of plant's adaptation strategy. The plant sensitivity exhibits developmentally regulated tissue or organ-specific remodeling which is genetically preserved for the superior fitness of the individual species. The molecular and functional characterization of different stimulus-response systems may thus open avenues for their future field applications.

\section{Acknowledgements}

Authors are thankful to the Department of Biotechnology (DBT), Government of India for the financial support under grant [BT/PR6044/AGII/106/863/2012] and research fellowship of DKP.

\section{Conflict of Interest}

The authors have no conflict of interest.

\section{References}

[1] Ding, Z., Galván-Ampudia, C.S., Demarsy, E., Łangowski, Ł., Kleine-Vehn, J., Fan, Y., Morita, M.T., Tasaka, M., Fankhauser, C., Offringa, R., et al. (2011) Light-Mediated Polarization of the PIN3 Auxin Transporter for the Phototropic Response in Arabidopsis. Nature Cell Biology, 13, 447-452. https://doi.org/10.1038/ncb2208

[2] Braam, J. (2005) In Touch: Plant Responses to Mechanical Stimuli. New Phytologist, 165, 373-389. https://doi.org/10.1111/j.1469-8137.2004.01263.x

[3] Morita, M.T. and Tasaka, M. (2004) Gravity Sensing and Signaling. Current Opinion in Plant Biology, 7, 712-718. https://doi.org/10.1016/j.pbi.2004.09.001

[4] Moriwaki, T., Miyazawa, Y., Kobayashi, A. and Takahashi, H. (2013) Molecular Mechanisms of Hydrotropism in Seedling Roots of Arabidopsis thaliana (Brassicaceae). American Journal of Botany, 100, 25-34. https://doi.org/10.3732/ajb.1200419

[5] Ho, C.M., Lee, Y.J., Kiyama, L.D., Dinesh-Kumar, S.P. and Liu, B. (2012) Arabidopsis Microtubule-Associated Protein MAP65-3 Cross-Links Antiparallel Microtubules toward Their plus Ends in the Phragmoplast via Its Distinct C-Terminal Microtubule Binding Domain. Plant Cell, 24, 2071-2085. https://doi.org/10.1105/tpc.111.092569

[6] Hofmeister, W.Z. (1863) Und berichtigungen zu den 1851 veröffentlichen untersuchungengen der entwicklung höherer krytogamen. Jahrbücher für Wissenschaftliche Botanik, 3, 259-293.

[7] Nakielski, J. (2008) The Tensor-Based Model for Growth and Cell Divisions of the Root Apex. I. The Signicance of Principal Directions. Planta, 228, 179-189. 
https://doi.org/10.1007/s00425-008-0728-y

[8] Robinson, S., Barbier, D.R.P., Chan, J., Bergmann, D., Prusinkiewicz, P. and Coen, E. (2011) Generation of Spatial Patterns through Cell Polarity Switching. Science, 333, 14361440. https://doi.org/10.1126/science.1202185

[9] Pietruszka, M. and Lewicka, S. (2007) Anisotropic Plant Growth Due to Phototropism. Journal of Mathematical Biology, 54, 45-55. https://doi.org/10.1007/s00285-006-0045-7

[10] Lewicka, S. and Pietruszka, M. (2007) Anisotropic Plant Cell Elongation Due to OrthoGravitropism. Journal of Mathematical Biology, 54, 91-100. https://doi.org/10.1007/s00285-006-0049-3

[11] Bichet, A., Desnos, T., Turner, S., Grandjean, O. and Höfte, H. (2001) BOTERO1 Is Required for Normal Orientation of Cortical Microtubules and Anisotropic Cell Expansion in Arabidopsis. The Plant Journal, 25, 137-148. https://doi.org/10.1046/j.1365-313x.2001.00946.x

[12] Yuen, C.Y.L., Sedbrook, J.C., Perrin, R.M., Carroll, K.L. and Masson, P.H. (2005) Loss-of-Function Mutations of Root Hair Defective3 Suppress Root Waving, Skewing, and Epidermal Cell File Rotation in Arabidopsis. Plant Physiology, 138, 701-714. https://doi.org/10.1104/pp.105.059774

[13] Chehab, E., Eich, E. and Braam, J. (2009) Thigmomorphogenesis: A Complex Plant Response to Mechano-Stimulation. Journal of Experimental Botany, 60, 43-56. https://doi.org/10.1093/jxb/ern315

[14] Kwon, Y., Kim, J.H., Nguyen, H.N., Jikumaru, Y., Kamiya, Y., Hong, S.-W. and Lee, H. (2013) A Novel Arabidopsis MYB-Like Transcription Factor, MYBH, Regulates Hypocotyl Elongation by Enhancing Auxin Accumulation. Journal of Experimental Botany, 64, 39113922. https://doi.org/10.1093/jxb/ert223

[15] De Smet, I. and Jurgens, G. (2007) Patterning the Axis in Plants-Auxin in Control. Current Opinion in Genetics \& Development, 17, 337-343. https://doi.org/10.1016/j.gde.2007.04.012

[16] Grieneisen, V.A., Xu, J., Maree, A.F.M., Hogeweg, P. and Scheres, B. (2007) Auxin Transport Is Sufficient to Generate a Maximum and Gradient Guiding Root Growth. Nature, 449, 1008-1013. https://doi.org/10.1038/nature06215

[17] Nishimura, T., Nakano, H., Hayashi, K.-i., Niwa, C. and Koshiba, T. (2009) Differential Downward Stream of Auxin Synthesized at the Tip has a Key Role in Gravitropic Curvature via TIR1/Afbs-Mediated Auxin Signaling Pathways. Plant and Cell Physiology, 50, 18741885. https://doi.org/10.1093/pcp/pcp129

[18] Chen, Q., Dai, X., De-Paoli, H., Cheng, Y., Takebayashi, Y., Kasahara, H., Kamiya, Y. and Zhao1, Y. (2014) Auxin Overproduction in Shoots Cannot Rescue Auxin Deficiencies in Arabidopsis Roots. Plant and Cell Physiology, 55, 1072-1079. https://doi.org/10.1093/pcp/pcu039

[19] Vanneste, S. and Friml, J.Í. (2009) Auxin: A Trigger for Change in Plant Development. Cell, 136, 1005-1016. https://doi.org/10.1016/j.cell.2009.03.001

[20] Friml, J. (2003) Auxin Transport-Shaping the Plant. Current Opinion in Plant Biology, 6, 7-12. https://doi.org/10.1016/S1369526602000031

[21] Esmon, C.A., Pedmale, U.V. and Liscum, E. (2005) Plant Tropisms: Providing the Power of Movement to a Sessile Organism. International Journal of Developmental Biology, 49, 665-674. https://doi.org/10.1387/ijdb.052028ce

[22] Inada, S., Ohgishi, M., Mayama, T., Okada, K. and Sakai, T. (2004) RPT2 Is a Signal Transducer Involved in Phototropic Response and Stomatal Opening by Association with Phototropin 1 in Arabidopsis thaliana. Plant Cell, 16, 887-896. https://doi.org/10.1105/tpc.019901 
[23] Friml, J., Yang, X., Michniewicz, M., Weijers, D., Quint, A., Tietz, O., Benjamins, R., et al. (2004) A PINOID-Dependent Binary Switch in Apical-Basal PIN Polar Targeting Directs Auxin Efflux. Science, 306, 862-865. https://doi.org/10.1126/science.1100618

[24] Rose, J., Braam, J., Fry, S. and Nishitani, K. (2002) The XTH Family of Enzymes Involved in Xyloglucan Endotransglucosylation and Endohydrolysis: Current Perspectives and a New Unifying Nomenclature. Plant \& Cell Physiology, 43, 1421-1435.

https://doi.org/10.1093/pcp/pcf171

[25] Fankhauser, C. and Christie, J.M. (2015) Plant Phototropic Growth. Current Biology, 25, R384-R389. https://doi.org/10.1016/j.cub.2015.03.020

[26] Hagen, G. and Guilfoyle, T. (2002) Auxin-Responsive Gene Expresion: Genes, Promoters and Regulatory Factors. Plant Molecular Biology, 49, 373-385.

https://doi.org/10.1023/A:1015207114117

[27] Liscum, E. and Reed, J. (2002) Genetics of AUX/IAA and ARF Action in Plant Growth and Development. Plant Molecular Biology, 49, 387-400.

https://doi.org/10.1023/A:1015255030047

[28] Peaucelle, A., Wightman, R. and Höfte, H. (2015) The Control of Growth Symmetry Breaking in the Arabidopsis Hypocotyl. Current Biology, 25, 1746-1752. https://doi.org/10.1016/j.cub.2015.05.022

[29] Prusinkiewicz, P. and Runions, A. (2012) Computational Models of Plant Development and Form. New Phytologist, 193, 549-569. https://doi.org/10.1111/j.1469-8137.2011.04009.x

[30] Sakai, T., Kagawa, T., Kasahara, M., Swartz, T.E., Christie, J.M., Briggs, W.R., M., W. and Okada, K. (2001) Arabidopsis Nph1 and Npl1: Blue Light Receptors That Mediate Both Phototropism and Chloroplast Relocation. Proceedings of the National Academy of Sciences of the United States of America, 98, 6969-6974.

https://doi.org/10.1073/pnas.101137598

[31] Blancaflor, E. and Masson, P. (2003) Plant Gravitropism. Unraveling the Ups and Downs of a Complex Process. Plant Physiology, 133, 1677-1690. https://doi.org/10.1104/pp.103.032169

[32] Fasano, J.M., Massa, G.D. and Gilroy, S. (2002) Ionic Signaling in Plant Responses to Gravity and Touch. Journal of Plant Growth Regulation, 21, 71-88.

https://doi.org/10.1007/s003440010049

[33] Fukaki, H., Fujisawa, H. and Tasaka, M. (1996) How Do Plant Shoots Bend up?-The Initial Step to Elucidate the Molecular Mechanisms of Shoot Gravitropism Using Arabidopsis thaliana. Journal of Plant Research, 109, 129-137 https://doi.org/10.1007/BF02344538

[34] Gilroy, S. (2008) Plant Tropisms. Current Biology, 18, R275-R277. https://doi.org/10.1016/j.cub.2008.02.033

[35] Kimbrough, J.M., Salinas-Mondragon, R., Boss, W.F., Brown, C.S. and Sederoff, H.W. (2004) The Fast and Transient Transcriptional Network of Gravity and Mechanical Stimulation in the Arabidopsis Rooot Apex. Plant Physiology, 136, 2790-2805. https://doi.org/10.1104/pp.104.044594

[36] Kiss, J.Z., Hertel, R. and Sack, F.D. (1989) Amyloplasts Are Necessary for Full Gravitropis Sensitivity in Roots of Arabidopsis thaliana. Planta, 177, 198-206. https://doi.org/10.1007/BF00392808

[37] Kolesnikov, Y.S., Kretynin, S.V., Volotovsky, I.D., Kordyum, E.L., Ruelland, E. and Kravets, V.S. (2015) Molecular Mechanisms of Gravity Perception and Signal Transduction in Plants. Protoplasma, 253, 987-1004. https://doi.org/10.1007/s00709-015-0859-5

[38] Perera, I.Y., Heilmann, I., Chang, S.C., Boss, W.F. and Kaufman, P.B. (2001) A Role for 
Inositol 1,4,5 Triphosphate in Gravitropic Signaling and the Retention of Cold-Perceived Gravistimulation of the Oat Shoot Pulvini. Plant Physiology, 125, 1499-1507.

https://doi.org/10.1104/pp.125.3.1499

[39] Zhang, S., Chen, S., Chen, F. and Jiang, B. (2011) The Role of Ionic Calcium in the Gravitropic Response of a Creeping Chrysanthemum Cultivar. Russian Journal of Plant Physiology, 58, 696-702. https://doi.org/10.1134/S1021443711040285

[40] Braam, J. and Davis, R.W. (1990) Rain-Induced, Wind-Induced and Touch-Induced Expression of Calmodulin and Calmodulin-Related Genes in Arabidopsis. Cell, 60, 357-364. https://doi.org/10.1016/0092-8674(90)90587-5

[41] Eapen, D., Barroso, M.L., Campos, M.E., Ponce, G., Corkidi, G., Dubrovsky, J.G. and Cassab, G.I. (2003) A No Hydrotropic Response Root Mutant That Responds Positively to Gravitropism in Arabidopsis. Plant Physiology, 131, 536-546.

https://doi.org/10.1104/pp.011841

[42] Takahashi, N., Yamazaki, Y., Kobayashi, A., Higashitani, A. and Takahashi, H. (2003) Hydrotropism Interacts with Gravitropism by Degrading Amyloplasts in Seedling Roots of Arabidopsis and Radish. Plant Physiology, 132, 805-810. https://doi.org/10.1104/pp.018853

[43] Baluška, F. and Mancuso, S. (2013) Root Apex Transition Zone as Oscillatory Zone. Frontiers in Plant Science, 4, 354. https://doi.org/10.3389/fpls.2013.00354

[44] Baluška, F. (2010) Recent Surprising Similarities between Plant Cells and Neurons. Plant Signaling \& Behavior, 5, 87-89. https://doi.org/10.4161/psb.5.2.11237

[45] Baluška, F., Mancuso, S., Volkmann, D. and Barlow, P.W. (2010) Root Apex Transition Zone: A Signalling-Response Nexus in the Root. Trends in Plant Science, 15, 402-408. https://doi.org/10.1016/j.tplants.2010.04.007

[46] Baluška, F. and Volkmann, D. (2011) Mechanical Aspects of Gravity-Controlled Growth, Development and Morphogenesis. In: Wojtaszek, P., Ed., Mechanical Integration of Plant Cells and Plants, Springer, Berlin, 195-223. https://doi.org/10.1007/978-3-642-19091-9_8

[47] Mancuso, S., Barlow, P.W., Volkmann, D. and Baluska, F. (2006) Actin Turnover-Mediated Gravity Response in Maize Root Apices: Gravitropism of Decapped Roots Implicates Gravisensing Outside of the Root Cap. Plant Signaling \& Behavior, 1, 52-58. https://doi.org/10.4161/psb.1.2.2432

[48] Baluška, F., Parker, J.S. and Barlow, P.W. (1992) Specific Patterns of Cortical and Endoplasmic Microtubules Associated with Cell Growth and Tissue Differentiation in Roots of Maize (Zea mays L.). Journal of Cell Science, 103, 191-200.

[49] Baluska, F. and Mancuso, S. (2013) Microorganism and Filamentous Fungi Drive Evolution of Plant Synapses. Frontiers in Cellular and Infection Microbiology, 3, 44. https://doi.org/10.3389/fcimb.2013.00044

[50] Dahlke, R.I., Luethen, H. and Steffens, B. (2009) ABP1. Plant Signaling \& Behavior, 5, 1-3. https://doi.org/10.4161/psb.5.1.10306

[51] Felle, H., Peters, W. and Palme, K. (1991) The Electrical Response of Maize to Auxins. Biochimica et Biophysica Acta, 1064, 199-204. https://doi.org/10.1016/0005-2736(91)90302-O

[52] Stéphanie, R., Jürgen, K.-V., Elke, B., Michael, S., Tomasz, P., Pawel, B., Steffen, V., Jing, Z., Sibu, S., Milada, Č., et al. (2010) ABP1 Mediates Auxin Inhibition of Clathrin-Dependent Endocytosis in Arabidopsis. Cell, 143, 111-121. https://doi.org/10.1016/j.cell.2010.09.027

[53] Xu, T., Wen, M., Nagawa, S., Fu, Y., Chen, J.-G., Wu, M.-J., Perrot-Rechenmann, C., Friml, J.Ã., Jones, A.M. and Yang, Z. (2010) Cell Surface- and Rho GTPase-Based Auxin Signaling Controls Cellular Interdigitation in Arabidopsis. Cell, 143, 99-110. https://doi.org/10.1016/j.cell.2010.09.003 
[54] Baluška, F., Samaj, J. and Menzel, D. (2003) Polar Transport of Auxin: Carrier-Mediated Flux across the Plasma Membrane or Neurotransmitter-Like Secretion? Trends in Cell Biology, 13, 282-285. https://doi.org/10.1016/S0962-8924(03)00084-9

[55] Tromas, A., Paponov, I. and Perrot-Rechenmann, C. (2010) Auxin Binding Protein 1: Functional and Evolutionary Aspects. Trends in Plant Science, 15, 436-446.

https://doi.org/10.1016/j.tplants.2010.05.001

[56] Łangowski, Ł., Růžička, K., Naramoto, S., Kleine-Vehn, J. and Friml, J. (2010) Trafficking to the Outer Polar Domain Defines the Root-Soil Interface. Current Biology, 20, 904-908. https://doi.org/10.1016/j.cub.2010.03.059

[57] Baluška, F., Volkmann, D. and Menzel, D. (2005) Plant Synapses: Actin-Based Domains for Cell-to-Cell Communication. Trends in Plant Science, 10, 106-111. https://doi.org/10.1016/j.tplants.2005.01.002

[58] Pelagio-Flores, R., Ortiz-Castro, R., Mendez-Bravo, A., Macias-Rodriguez, L. and Lopez-Bucio, J. (2011) Serotonin, a Tryptophan-Derived Signal Conserved in Plants and Animals, Regulates Root System Architecture Probably Acting as a Natural Auxin Inhibitor in Arabidopsis thaliana. Plant \& Cell Physiology, 52, 490-508. https://doi.org/10.1093/pcp/pcr006

[59] Jones, A.M. (1998) Auxin Transport: down and out and up Again. Science, 282, 2201-2202. https://doi.org/10.1126/science.282.5397.2201

[60] Friml, J. and Palme, K. (2002) Polar Auxin Transport-Old Questions and New Concepts? Plant Molecular Biology, 49, 273-284. https://doi.org/10.1023/A:1015248926412

[61] Friml, J., Wisniewska, J., Benkova, E., Mendgen, K. and Palme, K. (2002) Lateral Relocation of Auxin Efflux Regulator PIN3 Mediates Tropism in Arabidopsis. Nature, 415, 806-809. https://doi.org/10.1038/415806a

[62] Morelli, G. and Ruberti, I. (2000) Shade Avoidance Responses. Driving Auxin along Lateral Routes. Plant Physiology, 122, 621-626. https://doi.org/10.1104/pp.122.3.621

[63] Li, L., Xu, J., Xu, Z.-H. and Xue, H.-W. (2005) Brassinosteroids Stimulate Plant Tropisms through Modulation of Polar Auxin Transport in Brassica and Arabidopsis. Plant Cell, 17, 2738-2753. https://doi.org/10.1105/tpc.105.034397

[64] Massa, G. and Gilroy, S. (2003) Touch Modulates Gravity Sensing to Regulate the Growth of Primary Roots of Arabidopsis thaliana. Plant Journal, 33, 435-445.

https://doi.org/10.1046/j.1365-313X.2003.01637.x 
Submit or recommend next manuscript to SCIRP and we will provide best service for you:

Accepting pre-submission inquiries through Email, Facebook, LinkedIn, Twitter, etc. A wide selection of journals (inclusive of 9 subjects, more than 200 journals)

Providing 24-hour high-quality service

User-friendly online submission system

Fair and swift peer-review system

Efficient typesetting and proofreading procedure

Display of the result of downloads and visits, as well as the number of cited articles

Maximum dissemination of your research work

Submit your manuscript at: http://papersubmission.scirp.org/

Or contact ajps@scirp.org 